\title{
Two-Frequency Lasers: from Excess Quantum Noise to RF Photonics Applications
}

\author{
M. Alouini, F. Bretenaker, M. Brunel, D. Chauvat, \\ O. Emile, N.D. Lai, A. Le Floch, G. Ropars and M. Vallet \\ Laboratoire d'Electronique Quantique - Physique des Lasers \\ Unité Mixte de Recherche du Centre National de la Recherche Scientifique 6627 \\ Université de Rennes I, Campus de Beaulieu, 35042 Rennes Cedex, France
}

We illustrate the physics of two-frequency lasers by two examples. The first example illustrates the fundamental consequences of the existence of two eigenstates on the laser line width. We indeed show experimentally that the non-orthogonality of these two eigenstates results in an increase in the laser quantum noise. We also give a physical explanation of this vectorial excess noise factor. The second example illustrates the capabilities of two-frequency lasers in terms of applications. In the domain of RF frequency generation by optical means, we show how a pulsed two-frequency source can be built for lidar-radar applications and pulsed RF frequency generation.

PACS numbers: 42.55.Ah, 42.50.Ar, 42.50.Lc, 42.60.By, 42.60.Gd

\section{Introduction}

It is well known that the behavior of a laser can be described in terms of modes. In particular, the concepts of longitudinal and transverse modes are, when applicable, very helpful [1]. However, due to the fact that light is a transverse vibration, the vectorial nature of light must, in general, also be taken into account in the physics of lasers. In the case of quasi-monochromatic laser light, polarization phenomena can be easily described in the framework of the Jones matrix formalism [2]. The $z$-propagating light electric field is then described by a 2-vector $\boldsymbol{E}$ and any linear element can then be represented by a $2 \times 2$-matrix. In particular, if $\boldsymbol{M}$ is the Jones matrix for one round-trip inside the laser cavity starting from a given location, the laser light electric field $\boldsymbol{E}$ at this point must satisfy the eigenequation 
$\boldsymbol{M} \boldsymbol{E}=\lambda \boldsymbol{E}$, where $\lambda$ is the eigenvalue. This equation has two solutions: the so-called laser eigenstates [3]. In the general case, these two eigenstates have two different eigenvalues $\lambda_{1}$ and $\lambda_{2}$, two different eigenfrequencies $\nu_{1}$ and $\nu_{2}$, and two different eigenpolarizations represented by complex unit vectors $\hat{\boldsymbol{e}}_{1}$ and $\hat{\boldsymbol{e}}_{2}$. The existence of these two eigenstates has already led us to develop many applications based, e.g., on differential loss $\left(\left|\lambda_{1}\right| \neq\left|\lambda_{2}\right|\right)$ measurements (measurement of residual absorptions [4] or dichroisms [5]), on differential frequency $\left(\nu_{1} \neq \nu_{2}\right)$ measurements (polarimetry [6], magnetometry [7], or gyrometry [8] applications), or, for example, to the appearance of new dynamical regimes, such as pulsed regimes [9] or new mode-locking situations [10].

Here, we wish to illustrate two domains in which the existence of two different laser eigenstates has played a major role. In the first part of this paper, we show how the fact that the two laser eigenstates may be non-orthogonal $\left(\left\langle\hat{\boldsymbol{e}}_{1} \mid \hat{\boldsymbol{e}}_{2}\right\rangle \neq 0\right)$ modifies the quantum noise of the laser itself. In the second part, we will present one example illustrating the role that two-frequency lasers may, in the near future, play in the domain of RF photonics: the optical generation of pulsed microwaves.

\section{Vectorial excess noise factor}

The quantum noise-limited spectrum of a monomode laser is Lorentzian with a fundamental line width (FWHM) $\Delta \nu$ given by the famous Schawlow-Townes formula [11]:

$$
\Delta \nu=\frac{h \nu}{4 \pi} \frac{\Gamma^{2}}{P}
$$

where $h$ is Planck's constant, $\nu$ - the laser frequency, $\Gamma$ is the cold cavity decay rate, and $P$ - the optical power dissipated by the laser. Equations (1) has been obtained at the expense of many assumptions. One of them states that the gain atoms, which are responsible for both the stimulated emission which provides for gain and the spontaneous emission which adds the phase noise, obey the following equation:

$$
\frac{\text { spontaneous emission rate }}{\text { stimulated emission rate }}=\frac{1}{\langle n\rangle},
$$

where $\langle n\rangle$ is the average number of photons in the lasing mode. This is the famous "one photon per mode" paradigm, which is present in Einstein's equations. However, Eq. (2) is valid only if the lasing mode can be considered independently of the other modes of the field. In other words, Eq. (2) holds only if the laser modes constitute a complete set of orthogonal modes, upon which the laser field can be expanded, as is usually done before quantizing the electromagnetic field in a box [12]. However, this hypothesis is not always valid. This was pointed out by Siegman in 1989 in the case of the non-orthogonal transverse modes of an unstable cavity [13]. This has led to experimental demonstrations of increases in the laser 
line width of several orders of magnitude $[14,15]$. This effect, which is similar to Petermann's $K$ factor [16], has its counterpart in the case of the transverse modes of a stable cavity containing a diffracting aperture [17-19]. It also exists in the case of a cavity containing large losses, inducing a non-orthogonality of the longitudinal modes $[20,21]$. Here, we focus on the two eigenstates of an anisotropic cavity $[22,23]$.

\subsection{Semi-classical derivation of the vectorial excess noise factor}

Following Siegman [13] and van der Lee et al. [24], we derive here the excess noise factor using a semi-classical approach. Let us suppose that the laser state $|x(t)\rangle$ can be expanded upon the two (normalized) cold cavity eigenstates $\left|\hat{\boldsymbol{e}}_{1}\right\rangle$ and $\left|\hat{\boldsymbol{e}}_{2}\right\rangle$ :

$$
|x(t)\rangle=a_{1}(t)\left|\hat{\boldsymbol{e}}_{1}\right\rangle+a_{2}(t)\left|\hat{\boldsymbol{e}}_{2}\right\rangle .
$$

The equation of evolution of the laser is given by

$$
\frac{\mathrm{d}}{\mathrm{d} t}|x(t)\rangle=N|x(t)\rangle+|l(t)\rangle
$$

The first term on the left hand side of Eq. (4) is the deterministic evolution of the laser field, where $\boldsymbol{N}$ is related to the Jones matrix $\boldsymbol{M}$ for one round-trip inside the cavity by

$$
N=\frac{c}{2 L}(\boldsymbol{M}-\mathbf{1}),
$$

with $c$ the velocity of light and $L$ the cavity length. $\boldsymbol{N}$ has the same eigenstates as $\boldsymbol{M}$, but with the eigenvalues $\mu_{i}(i=1,2)$ related to those $\left(\lambda_{i}\right)$ of $\boldsymbol{M}$ by

$$
\mu_{i}=\frac{c}{2 L}\left(\lambda_{i}-1\right)
$$

The second term $|l(t)\rangle$ in Eq. (4) is a $\delta$-correlated Langevin noise which holds for spontaneous emission. In the general case where the two eigenstates are not orthogonal, it is worth introducing the adjoints $\left|f_{1}\right\rangle$ and $\left|f_{2}\right\rangle$ of the eigenstates $\left|\hat{\boldsymbol{e}}_{1}\right\rangle$ and $\left|\hat{\boldsymbol{e}}_{2}\right\rangle$ defined by

$$
\left\langle\boldsymbol{f}_{1} \mid \hat{\boldsymbol{e}}_{2}\right\rangle=\left\langle\boldsymbol{f}_{2} \mid \hat{\boldsymbol{e}}_{1}\right\rangle=0
$$

which we normalize so that

$$
\left\langle f_{1} \mid \hat{e}_{1}\right\rangle=\left\langle f_{2} \mid \hat{e}_{2}\right\rangle=1 .
$$

Equation (4) can then be projected on $\left\langle f_{i}\right|$, leading to

$$
\frac{\mathrm{d}}{\mathrm{d} t} a_{i}(t)=\mu_{i} a_{i}(t)+\left\langle f_{i} \mid l(t)\right\rangle
$$

which has the following formal solution:

$$
a_{i}(t)=a_{i}(0) \mathrm{e}^{\mu_{i} t}+\int_{0}^{t} \mathrm{~d} t^{\prime} \mathrm{e}^{\mu_{i}\left(t-t^{\prime}\right)}\left\langle f_{i} \mid l\left(t^{\prime}\right)\right\rangle .
$$


Let us now suppose that for $t \rightarrow \infty$ the laser oscillates in eigenstate 1 only. Then the laser state reads

$$
|x(t)\rangle=a_{1}(0) \mathrm{e}^{\mu_{1} t}\left|\hat{\boldsymbol{e}}_{1}\right\rangle+\int_{0}^{t} \mathrm{~d} t^{\prime} \mathrm{e}^{\mu_{1}\left(t-t^{\prime}\right)}\left\langle f_{1} \mid l\left(t^{\prime}\right)\right\rangle\left|\hat{\boldsymbol{e}}_{1}\right\rangle .
$$

The first term of this expression is the noise-free laser evolution. The second term is the laser quantum noise. To derive the power spectral density of this term, we have to calculate the Fourier transform of its autocorrelation. If we suppose that the Langevin noise $|l(t)\rangle$ is $\delta$-correlated and is also isotropic, then this noise power is proportional to the following factor:

$$
K=\left\langle f_{1} \mid f_{1}\right\rangle .
$$

The adjoint mode $\left|f_{1}\right\rangle$ is then expanded upon the eigenstates $\left|\hat{\boldsymbol{e}}_{1}\right\rangle$ and $\left|\hat{\boldsymbol{e}}_{2}\right\rangle$ :

$$
\left|f_{1}\right\rangle=\alpha_{1}\left|\hat{\boldsymbol{e}}_{1}\right\rangle+\beta_{1}\left|\hat{\boldsymbol{e}}_{2}\right\rangle
$$

Equation (13) can be projected upon $\left|\hat{\boldsymbol{e}}_{1}\right\rangle$ and $\left|\hat{\boldsymbol{e}}_{2}\right\rangle$, leading to

$$
\begin{aligned}
& 1=\alpha_{1}+\beta_{1}\left\langle\hat{\boldsymbol{e}}_{1} \mid \hat{\boldsymbol{e}}_{2}\right\rangle, \\
& 0=\alpha_{1}\left\langle\hat{\boldsymbol{e}}_{2} \mid \hat{\boldsymbol{e}}_{1}\right\rangle+\beta_{1} .
\end{aligned}
$$

$\alpha_{1}$ and $\beta_{1}$ can then be eliminated, and (12) and (13) lead to

$$
K=\frac{1}{1-\left|\left\langle\hat{\boldsymbol{e}}_{1} \mid \hat{\boldsymbol{e}}_{2}\right\rangle\right|^{2}} .
$$

This expression shows that $K>1$. This so-called excess noise factor is then predicted to enhance the laser line width as soon as the eigenstates are not orthogonal, as is now going to be proved.

\subsection{Observation of the vectorial excess noise factor}

Let us consider the cavity of Fig. 1, built with an active medium and two mirrors $M_{1}$ and $M_{2}$. It contains a phase anisotropy aligned with the $x$ and $y$ axes (retardance $\phi$ ) and a loss anisotropy $\Delta t_{\perp}$. The axes of this partial polarizer are aligned at $\pm 45^{\circ}$ from $x$ and $y$. We call $\alpha(0 \leq \alpha \leq 1)$ the ratio between the field

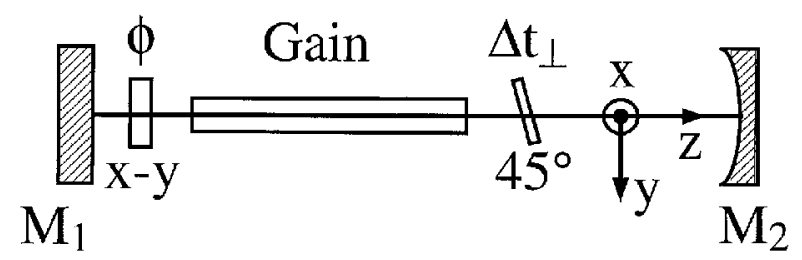

Fig. 1. Non-Hermitian cavity. $M_{1}$ and $M_{2}-$ mirrors, $\phi-x-y$ phase anisotropy, $\Delta t_{\perp}$ - loss anisotropy oriented at $45^{\circ}$ of the $x$ and $y$ axes. 


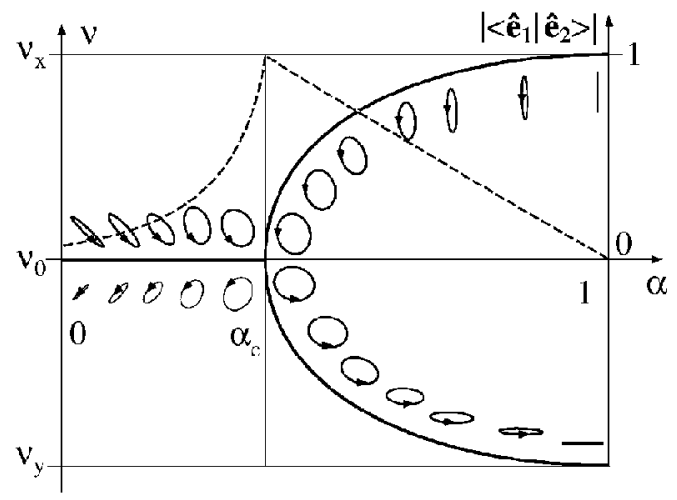

Fig. 2. Full line: variation of the eigenfrequencies $\nu_{1}$ and $\nu_{2}$ of the laser cavity versus loss anisotropy $\alpha$. The corresponding polarization ellipses are also schematized. Dashed line: evolution of the modulus of the scalar product of the two eigenstates versus $\alpha$.

transmission coefficients along the high and low loss axes of $\Delta t_{\perp}$. Then the Jones matrix $\boldsymbol{M}$ for one round-trip inside this cavity reads

$$
\boldsymbol{M}=\frac{1}{2}\left[\begin{array}{cc}
\left(\alpha^{2}+1\right) \mathrm{e}^{\mathrm{i} \phi} & \alpha^{2}-1 \\
\alpha^{2}-1 & \left(\alpha^{2}+1\right) \mathrm{e}^{-\mathrm{i} \phi}
\end{array}\right] .
$$

This matrix is non-Hermitian. Two different situations must be considered, as can be seen in Fig. 2. First, for $\alpha<\alpha_{c}=\left[\left(1-\sin ^{2} \phi\right) /\left(1+\sin ^{2} \phi\right)\right]^{1 / 2}$, i.e., when the loss anisotropy prevails over the phase anisotropy, the two eigenfrequencies of the laser cavity are degenerate. The corresponding eigenstates are elliptically polarized, with their axes roughly aligned with the loss anisotropy axes. Of course, one of these eigenstates experiences more losses than the other one, and may thus not oscillate. On the contrary, for $\alpha>\alpha_{\mathrm{c}}$, the phase anisotropy is large enough to raise the degeneracy between the two eigenfrequencies $\nu_{1}$ and $\nu_{2}$ (see Fig. 2). The corresponding eigenstates are elliptically polarized, with their axes approximately aligned with $x$ and $y$, i.e., the axes of the phase anisotropy, and have the same losses. Figure 2 summarizes the variations of the eigenfrequencies $\nu_{1}$ and $\nu_{2}$ versus the loss anisotropy $\alpha$, together with the corresponding polarizations. One can notice that the eigenstates are almost never orthogonal. This is also stressed by the dashed curve of Fig. 2, which represents the variations of the modulus of the scalar product of the two normalized eigenvectors $\hat{\boldsymbol{e}}_{1}$ and $\hat{\boldsymbol{e}}_{2}$ versus $\alpha$. From Eq. (15), the expected excess noise factor is then given by

$$
K=\frac{\left(1-\alpha^{2}\right)^{2}}{\left(1-\alpha^{2}\right)^{2}-\left(1+\alpha^{2}\right)^{2} \sin ^{2} \phi}
$$

in the region where both frequencies are degenerate $\left(\alpha<\alpha_{c}\right)$, and by

$$
K=\frac{\left(1+\alpha^{2}\right)^{2} \sin ^{2} \phi}{\left(1+\alpha^{2}\right)^{2} \sin ^{2} \phi-\left(1-\alpha^{2}\right)^{2}}
$$

in the region where the eigenfrequencies are different $\left(\alpha>\alpha_{\mathrm{c}}\right)$. 


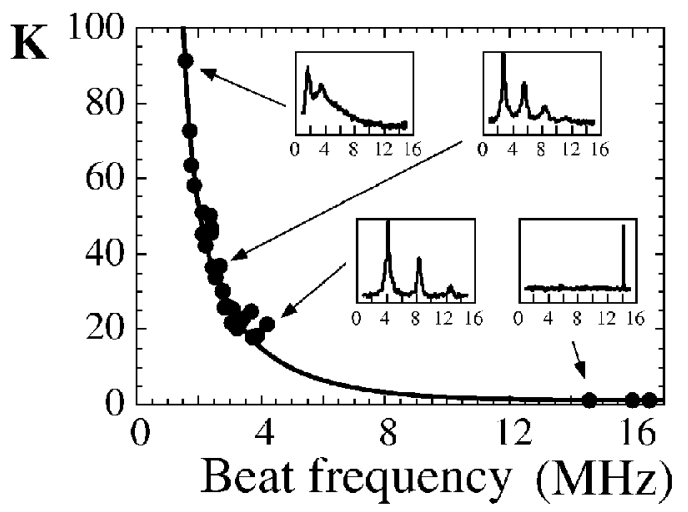

Fig. 3. Experimental (dots) and theoretical (full line) evolutions of the excess noise factor $K$ versus the beat frequency between the two eigenstates. The inserts reproduce the spectra of the beat notes corresponding to several typical situations (logarithmic vertical scale; horizontal axis: 0 to $16 \mathrm{MHz}$ ).

To measure this excess noise factor, we have built a $27 \mathrm{~cm}$ long He-Xe laser oscillating at $3.51 \mu \mathrm{m}$. This transition exhibits a large gain, and allows the two eigenstates to oscillate simultaneously for $\alpha>\alpha_{c}$, i.e., when the two eigenstates have the same losses. The laser line width can then simply be measured by recording the spectrum of the beat note between these two eigenstates and by observing its line width.

We choose a phase retardance $\phi$ corresponding to a beat note $(c / 2 L) \phi / \pi=$ $16 \mathrm{MHz}$ in the absence of any loss anisotropy. Some typical beat note spectra obtained for different values of the loss anisotropy $\alpha$ are reproduced as inserts in Fig. 3. Such spectra lead to the measurements of $K$ summarized as black dots in Fig. 3. The measured enhancement of the laser line width, reaching values as high as 92, is in close agreement with Eq. (18) (full line in Fig. 3).

\subsection{Adjoint coupling}

The preceding results have been obtained only for the part of Fig. 2 where $\alpha>\alpha_{\mathrm{c}}$, i.e., where the two eigenstates can be made to oscillate simultaneously. However, the opposite situation, i.e., when $\alpha<\alpha_{c}$ and $\left|\lambda_{1}\right|>\left|\lambda_{2}\right|$, can provide us with some physical insight in the physics of non-Hermitian cavities. To this aim, we built the experiment of Fig. 4, in which a monomode $633 \mathrm{~nm}$ laser beam is injected inside a passive cavity similar to the one of Fig. 1 . This cavity is now $L=48 \mathrm{~cm}$ long. It also contains an aperture to get rid of high-order transverse modes. Mirror $M_{2}$ is mounted on a piezoelectric element, thus allowing us to modulate the phase $\delta$ undergone by light during one round-trip inside this cavity. Let us consider the reflection operator for this cavity 


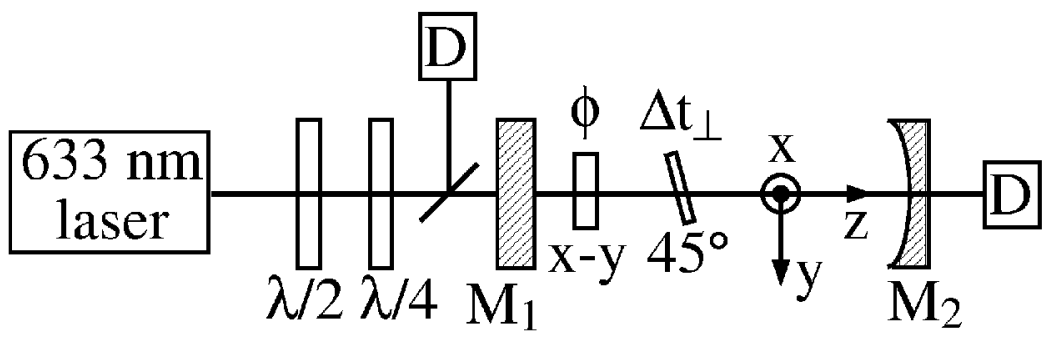

Fig. 4. Injection of a monomode laser inside a non-Hermitian passive cavity. The values of the experimental parameters are $L=48 \mathrm{~cm}, \phi=7^{\circ}, \alpha=0.854<\alpha_{\mathrm{c}}, t^{2}=0.897, r_{1}^{2}=$ $r_{2}^{2}=0.985$, and $t_{1}^{2}=t_{2}^{2}=0.012$ ( $\mathrm{D}$ denotes light detectors).

$$
\boldsymbol{R}=-r_{1}+t_{1}^{2} r_{2} t^{2} \mathrm{e}^{\mathrm{i} \delta} \boldsymbol{M} \sum_{n=0}^{\infty}\left(r_{1} r_{2} t^{2} \mathrm{e}^{\mathrm{i} \delta} \boldsymbol{M}\right)^{n}=-r_{1}+\frac{t_{1}^{2} r_{2} t^{2} \mathrm{e}^{\mathrm{i} \delta} \boldsymbol{M}}{1-r_{1} r_{2} t^{2} \mathrm{e}^{\mathrm{i} \delta} \boldsymbol{M}},
$$

where $r_{1}$ and $r_{2}$ (respectively $t_{1}$ and $t_{2}$ ) are the field reflection (respectively transmission) coefficients of the cavity mirrors, $t$ holds for the isotropic losses for one pass through the cavity (tilted plate and diffracting aperture), and $M$ is given by Eq. (16). The operator $\boldsymbol{R}$ has the same eigenvectors $\hat{\boldsymbol{e}}_{1}$ and $\hat{\boldsymbol{e}}_{2}$ as $\boldsymbol{M}$, but with different eigenvalues $\rho_{1}$ and $\rho_{2}$. The half-wave and quarter-wave plates located between the laser and the cavity can be rotated in order to create any incident polarization state $|\boldsymbol{u}\rangle$. To compute the intensity reflected by the cavity, we expand this normalized arbitrary state upon the adjoint modes

$$
|u\rangle=\left\langle f_{1} \mid u\right\rangle\left|\hat{\boldsymbol{e}}_{1}\right\rangle+\left\langle f_{2} \mid \boldsymbol{u}\right\rangle\left|\hat{\boldsymbol{e}}_{2}\right\rangle
$$

leading to

$$
\begin{aligned}
& \left\langle u\left|\boldsymbol{R}^{\dagger} \boldsymbol{R}\right| u\right\rangle=\left|\left\langle f_{1} \mid u\right\rangle\right|^{2}\left|\rho_{1}\right|^{2}+\left|\left\langle f_{2} \mid u\right\rangle\right|^{2}\left|\rho_{2}\right|^{2} \\
& +2 \Re\left\{\left(u\left|f_{1}\right\rangle\left\langle f_{2} \mid u\right\rangle \rho_{1}^{*} \rho_{2}\left\langle\hat{\boldsymbol{e}}_{1} \mid \hat{\boldsymbol{e}}_{2}\right\rangle\right\} .\right.
\end{aligned}
$$

In the case where the two eigenstates are orthogonal $\left(\left\langle\hat{\boldsymbol{e}}_{1} \mid \hat{\boldsymbol{e}}_{2}\right\rangle=0\right)$, the last term of Eq. (21) vanishes, and the following inequality holds:

$$
\left|\rho_{1}\right|^{2} \leq\left\langle u\left|\boldsymbol{R}^{\dagger} \boldsymbol{R}\right| u\right\rangle \leq\left|\rho_{2}\right|^{2}
$$

This means that the output light cannot be coupled inside the cavity less efficiently than the high-loss eigenstate and more efficiently than the low-loss eigenstate.

On the contrary, when the two eigenstates are not orthogonal, this inequality is no longer valid, as shown in Fig. 5. In Fig. 5a, the cavity reflection coefficient has been computed versus $\delta$ for $\phi=7^{\circ}, \alpha=0.854<\alpha_{c}, t^{2}=0.897, r_{1}^{2}=r_{2}^{2}=0.985$, and $t_{1}^{2}=t_{2}^{2}=0.012$. These parameters correspond to the experimental values. The two curves labeled 1 and 2 in Fig. 5a correspond to the low- and high-loss eigenstates respectively. We then look for the input polarizations that minimize and maximize the reflection coefficient of the cavity at resonance. This leads to 

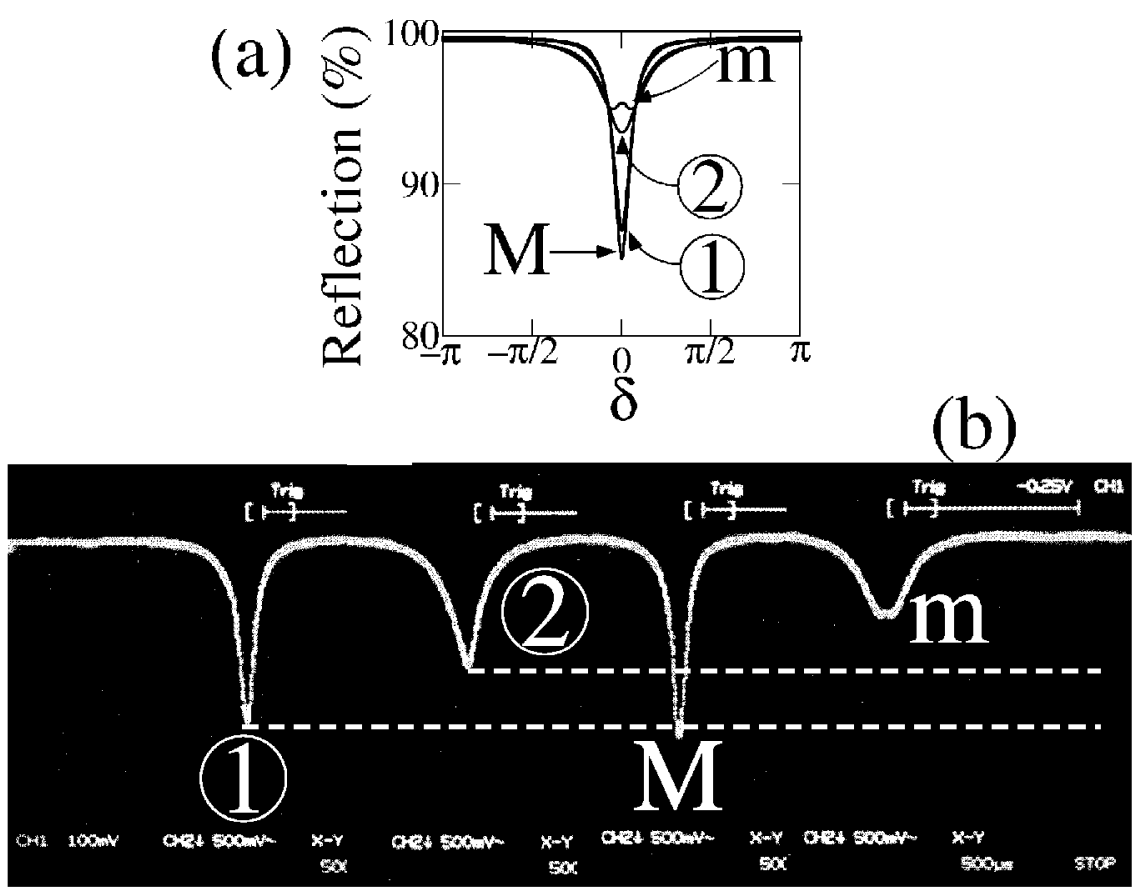

Fig. 5. (a) Theoretical evolution of the cavity reflection coefficients versus round-trip phase $\delta$ for injection of the eigenstates 1 and 2 and for the polarizations $m$ and $M$ that maximize the reflection of the cavity at resonance. (b) Corresponding experimental results. The input states of polarization are created according to the ones calculated in $(\mathrm{a})$.

the curves labeled $M$ and $m$, respectively, in Fig. 5a, which are actually obtained for the polarizations $f_{1}$ and $f_{2}$ of the adjoint modes, respectively.

These computed polarization states are then created experimentally using the half-wave and quarter-wave plates located at the output of the laser. We then obtain the corresponding experimental signals of Fig. 5b, which clearly show that the light coupled inside the cavity with the adjoint polarization labeled $M$ is more important than with the low-loss eigenstate labeled 1 . Conversely, polarization $m$ is better reflected by the cavity than the low-loss eigenstate labeled 2.

This result is even more striking in the case of the transmission operator (see Fig. 6). Here again, the input polarization can be tailored to reach coupling efficiencies larger than for the low-loss eigenstate and smaller than for the low-loss eigenstate, thus illustrating the non-Hermiticity of this cavity*.

The results of Figs. 5 and 6 show simply that, in non-Hermitian cavities, the

*The eigenstates of the transmission operator are slightly different from those of $\boldsymbol{M}$ and $\boldsymbol{R}$, hence leading to small deviations of the states of polarization which minimize and maximize the transmission with respect to the adjoint modes. 
(a)
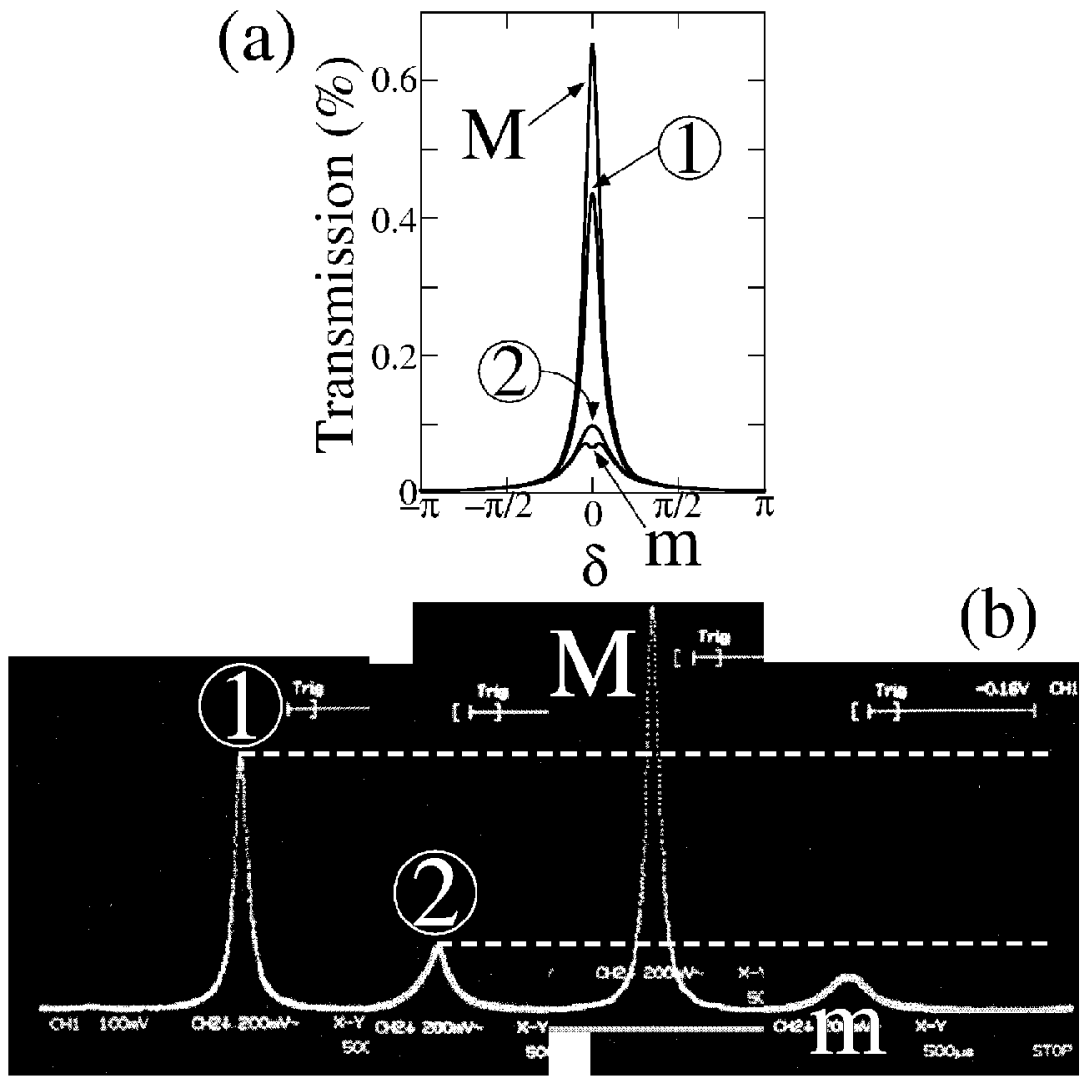

Fig. 6. Same as Fig. 5 for the transmission operator.

usual intuitive approach to photon problems fails. Indeed, they show that a one photon state incident on this cavity can apparently lead to more than one photon inside the cavity, provided that this photon is "adjoint coupled", i.e., is polarized along the adjoint mode of the low-loss state [25-27]. This explains physically how the vacuum fluctuations are more efficiently coupled inside a non-Hermitian cavity than stated by the usual "one photon per mode" rule, hence increasing the laser quantum noise.

\section{Pulsed RF lasers}

Recently, there has been a great interest in the optical generation of microwaves, from the $\mathrm{GHz}$ to the $\mathrm{THz}$ range [28]. This interest has been triggered by both the potential applications in the domains of telecommunications or remote sensing, and simply by the fact that no sources are available in some of these spectral regions. This has stimulated many developments, in particular in our group where we use the two eigenstates of a laser with $\nu_{1} \neq \nu_{2}$ to create the beat note $\nu_{1}-\nu_{2}$. Since some applications of two-frequency lasers (such as lidar-radar de- 
vices) require to reach higher peak powers, we here focus on the realization of a pulsed two-frequency laser [29].

\subsection{Principle of pulsed two-frequency operation}

Let us consider the laser cavity of Fig. 7, whose propagation axis is $z$. It is closed with mirrors $M_{1}$ and $M_{2}$ separated by $L=9.7 \mathrm{~cm}$ and contains an isotropic active medium (a diode pumped Nd:YAG crystal) and a linear phase anisotropy creating a retardance $\phi$ between the $x$ and $y$ polarizations. It also contains a $\mathrm{Cr}^{4+}$ :YAG saturable absorber (passive $Q$-switch) whose absorbing dipoles are aligned along the [100], [010], and [001] crystallographic axes. In the linear regime (small signal) the absorption is isotropic. Then a Jones matrix analysis for the cold cavity yields two linearly polarized eigenstates aligned along the $x$ and $y$ axes with a frequency difference $\Delta \nu=(c / 2 L) \phi / \pi$. The saturable absorber aims at turning this two-frequency laser into a pulsed two-frequency laser, i.e., into a laser emitting pulses, each of them containing the two frequencies. Two particular

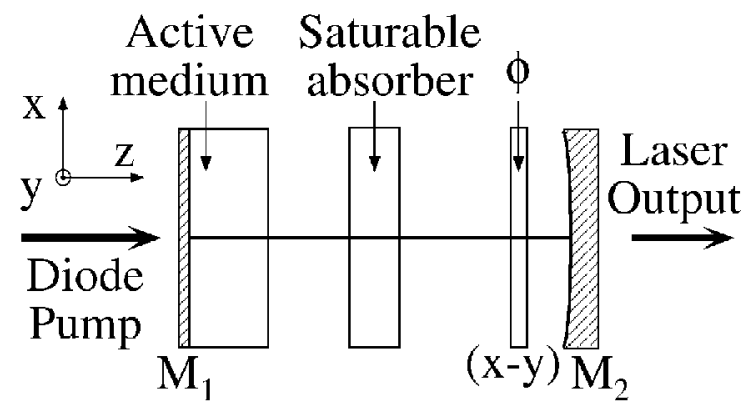

Fig. 7. Pulsed two-frequency laser. The $L=4.7 \mathrm{~cm}$ long cavity contains a diode-pumped Nd:YAG active medium. The saturable absorber is a $\mathrm{Cr}^{4+}: \mathrm{YAG}_{\text {cys- }}$ tal which aims at passively $Q$-switching the laser. The phase retardance $\phi$ creates the two eigenstates with their two frequencies.
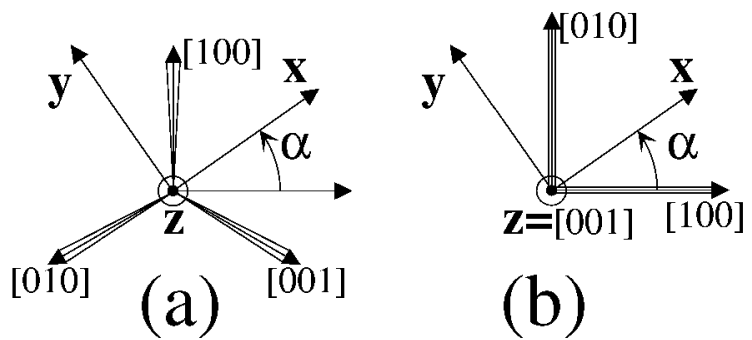

Fig. 8. The absorbing dipoles are oriented along the [100], [010], and [001] directions and the laser eigenstates along the $x$ and $y$ directions. (a) [111]-cut $\mathrm{Cr}^{4+}: Y A G$, (b) [001]-cut $\mathrm{Cr}^{4+}: \mathrm{YAG}$. 
situations must be emphasized, corresponding to two different orientations of the $\mathrm{Cr}^{4+}$ :YAG samples (see Fig. 8): the $z \|[111]$ orientation (see Fig. 8a) and the $z \|$ [001] orientation (see Fig. 8b). The saturation of the three absorber species depends strongly on the orientation of the $x$ and $y$ directions of the eigenstates with respect to the crystallographic axes of the absorber. We thus expect the laser to behave in different manners depending on the orientation of the absorbing dipoles with respect to the polarizations $x$ and $y$ of the two eigenstates. Indeed, one can
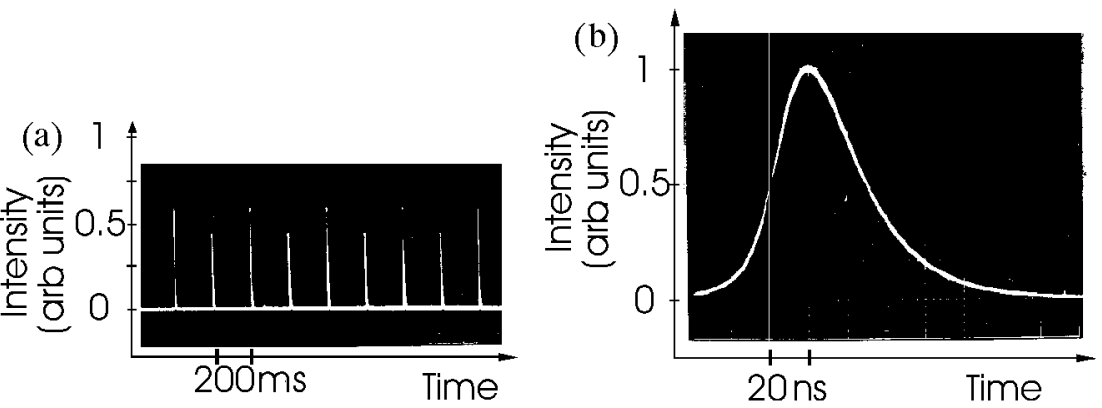

Fig. 9. Experimental results obtained with the [111]-cut sample. (a) Pulse train observed through a linear polarizer: the two eigenstates are emitted successively. (b) Typical pulse.
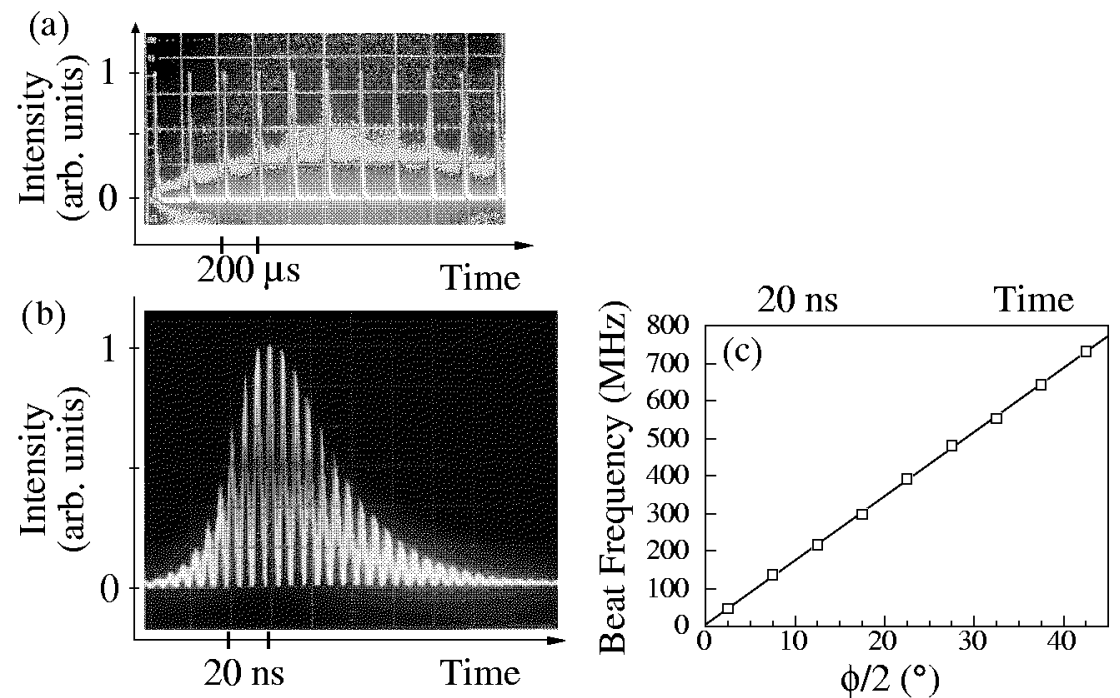

Fig. 10. Experimental results obtained with the [001]-cut sample with $\alpha=45^{\circ}$. (a) Pulse train observed through a linear polarizer oriented at $45^{\circ}$ with respect to the $x$ and $y$ directions: the two eigenstates are emitted simultaneously. (b) Typical pulse containing the beat note between the two eigenstates. (c) Experimental evolution of the beat frequency versus intracavity birefringence. 
see in Fig. 8a that in the case of the [111]-cut crystal, the situation is always non-symmetrical for any value of angle $\alpha$. For example, in the case of Fig. 8a, the $x$-polarized eigenstate interacts mostly with the [010]-oriented dipoles while the $y$-polarized eigenstate interacts mostly with the [001]-oriented dipoles. This means that the two eigenstates do not have to cooperate to saturate their absorptions. This results experimentally in the behavior of Fig. 9: the laser emits a train of pulses which are alternatively emitted along the two eigenstates. Hence, the laser light does not contain both eigenstates simultaneously and the pulsed two-frequency regime is not obtained.

We hence turn to the other orientation of the $\mathrm{Cr}^{4+}$ :YAG crystal, namely $z \|$ [001]. Then, as can be seen from Fig. 8b, a symmetric situation can be reached for $\alpha=45^{\circ}$. In this case, the two eigenstates interact equally with the [100] and [010] dipole classes. These two eigenstates then have to cooperate to saturate the losses, leading to the result of Fig. 10: the pulses now contain the two frequencies and the beat note at $\Delta \nu$ can be seen in Fig. $10 \mathrm{~b}$. This beat frequency can be tuned between 0 and $c / 4 L=770 \mathrm{MHz}$, as can be seen in Fig. $10 \mathrm{c}$.

\subsection{Towards higher beat frequencies}

Laser ranging and lidar-radar applications require the use of beat frequencies higher than $1 \mathrm{GHz}$ and longer pulse durations. Indeed, in these applications, the measurement is based on the Doppler effect of the RF modulation of the retroreflected pulse. The beat note frequency should consequently be fast enough with
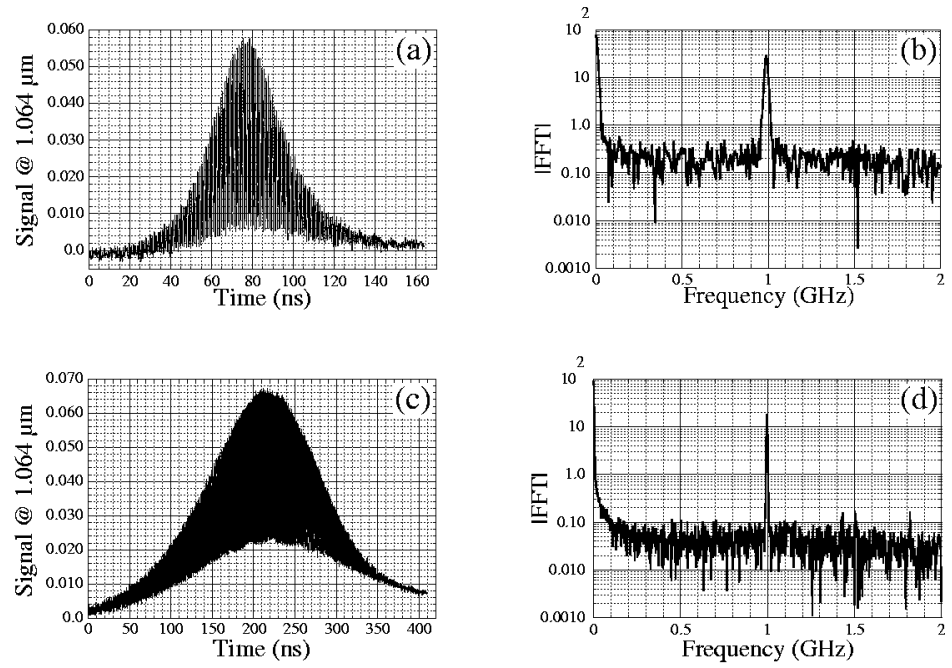

Fig. 11. Experimental (a), (c) pulses and (b), (d) beat note spectra obtained with a $2.8 \mathrm{~cm}$ long laser. The pulse duration can be adjusted without changing the beat note frequency. 
a good spectral resolution (long pulse duration). We hence now turn to a shorter cavity $(L=2.8 \mathrm{~cm}$ ) with a different pumping architecture. This laser allows us to reach beat frequencies larger than $2 \mathrm{GHz}$. Figure 11a shows a typical pulse obtained from this laser with a $1 \mathrm{GHz}$ beat note. This pulse is $36 \mathrm{~ns}$ long (FWHM) and its spectrum is reproduced in Fig. 11b. With a $800 \mathrm{~mW}$ pump power, the repetition rate is $13 \mathrm{kHz}$ and the pulse energy $0.2 \mu \mathrm{J}$.

One particularly interesting aspect of the pulsed two-frequency laser that we have built is that the pulse duration can be adjusted by changing the geometries of the cavity and/or of the pumping without modifying the beat note frequency. This can be seen in Fig. 11c, where the pulse duration is now as long as $150 \mathrm{~ns}$ and where the $1 \mathrm{GHz}$ beat note is still present. The corresponding spectrum is displayed in Fig. 11d. The usefulness of such long two-frequency pulses in the domain of lidar-radar applications is now under active investigation [30].

\section{Conclusion}

We have seen that the simple fact that light is a transverse vibration leads in general to the existence of two eigenstates in lasers. Such eigenstates have been used here (i) to reveal part of the physics underlying the appearance of an excess quantum noise factor in the laser line width and (ii) to build pulsed two-frequency lasers for lidar-radar applications. These examples are only a very small part of the potentialities of such lasers. In particular, two-frequency lasers are now actively investigated for telecommunications [31] and spatial heterodyne spectroscopy applications.

\section{Acknowledgments}

Part of this work has been supported by the Délégation Générale à l'Armement, the Réseau National de Recherche en Télécommunications, the Action Concertée Incitative Photonique, and the Conseil Régional de Bretagne. We thank L. Morvan, D. Dolfi, and J.-P. Huignard for their constant interest in this work.

\section{References}

[1] A.E. Siegman, Lasers, University Science, Mill Valley, CA 1986.

[2] R.C. Jones, J. Opt. Soc. Am. 31, 488 (1941); H. Hurwitz, Jr., R.C. Jones, J. Opt. Soc. Am. 31, 493 (1941); R.C. Jones, J. Opt. Soc. Am. 31, 500 (1941); R.C. Jones, J. Opt. Soc. Am. 32, 486 (1942).

[3] A. Le Floch, Doctorat d'Etat, University of Rennes, Rennes 1977, and references therein.

[4] N.H. Tran, D. Jacob, A. Le Floch, F. Bretenaker, Electron. Lett. 30, 2026 (1994).

[5] F. Bretenaker, A. Le Floch, L. Dutriaux, Phys. Rev. Lett. 68, 931 (1992). 
[6] M. Vallet, F. Bretenaker, A. Le Floch, R. Le Naour, M. Oger, Opt. Commun. 168, 423 (1999).

[7] F. Bretenaker, B. Lépine, J.-C. Cotteverte, A. Le Floch, Phys. Rev. Lett. 69, 909 (1992)

[8] M. Vallet, N.H. Tran, P. Tanguy, A. Le Floch, F. Bretenaker, Opt. Lett. 19, 1219 (1994).

[9] M. Brunel, O. Emile, M. Alouini, A. Le Floch, F. Bretenaker, Opt. Lett. 24, 229 (1999).

[10] M. Vallet, M. Brunel, F. Bretenaker, M. Alouini, A. Le Floch, G.P. Agrawal, Appl. Phys. Lett. 74, 3266 (1999).

[11] A.L. Schawlow, C.H. Townes, Phys. Rev. 112, 1940 (1958).

[12] See for example M. Sargent III, M.O. Scully, W.E. Lamb, Jr., Laser Physics, Addison-Wesley, Reading, MA 1974.

[13] A.E. Siegman, Phys. Rev. A 39, 1253 (1989); Phys. Rev. A 39, 1264 (1989).

[14] Y.J. Cheng, C.G. Fanning, A.E. Siegman, Phys. Rev. Lett. 77, 627 (1996).

[15] M.A. van Eijkelenborg, A.M. Lindberg, M.S. Thijssen, J.P. Woerdman, Phys. Rev. Lett. 77, 4314 (1996).

[16] K. Petermann, IEEE J. Quantum Electron. QE-15, 566 (1979).

[17] M. Brunel, G. Ropars, A. Le Floch, F. Bretenaker, Phys. Rev. A 55, 4563 (1997).

[18] Å.M. Lindberg, M.A. van Eijkelenborg, K. Joosten, G. Nienhuis, J.P. Woerdman, Phys. Rev. A 57, 3036 (1998).

[19] O. Emile, M. Brunel, F. Bretenaker, A. Le Floch, Phys. Rev. A 57, 4889 (1998).

[20] W.A. Hamel, J.P. Woerdman, Phys. Rev. A 40, 2785 (1989).

[21] W.A. Hamel, J.P. Woerdman, Phys. Rev. Lett. 64, 1506 (1990).

[22] A.M. van der Lee, N.J. van Druten, A.L. Mieremet, M.A. van Eijkelenborg, Å.M. Lindberg, M.P. van Exter, J.P. Woerdman, Phys. Rev. Lett. 79, 4357 (1997).

[23] O. Emile, M. Brunel, A. Le Floch, F. Bretenaker, Europhys. Lett. 43, 153 (1998).

[24] A.M. van der Lee, M.P. van Exter, A.L. Miemeret, N.J. van Druten, J.P. Woerdman, Phys. Rev. Lett. 81, 5121 (1998).

[25] A.E. Siegman, Appl. Phys. B 60, 247 (1995).

[26] A.E. Siegman, in: Coherence and Quantum Optics, Vol. 7, Eds. J.H. Eberly, L. Mandel, E. Wolf, Plenum Press, New York 1996, p. 229.

[27] O. Emile, D. Chauvat, A. Le Floch, F. Bretenaker, Opt. Lett. 24, 22 (1999).

[28] See for example M. Alouini, F. Bretenaker, M. Brunel, D. Chauvat, O. Emile, A. Le Floch, G. Ropars, M. Vallet, Proc. SPIE 4353, 145 (2001).

[29] M. Brunel, O. Emile, M. Vallet, F. Bretenaker, A. Le Floch, L. Fulbert, J. Marty, B. Ferrand, E. Molva, Phys. Rev. A 60, 4052 (1999).

[30] L. Morvan, N.D. Lai, D. Dolfi, M. Brunel, F. Bretenaker, A. Le Floch, in preparation.

[31] M. Alouini, B. Benazet, M. Vallet, M. Brunel, P. Di Bin, F. Bretenaker, A. Le Floch, IEEE Photon. Tech. Lett. 13, 367 (2001). 